\title{
Intrahippocampal Infusions of K-ATP Channel Modulators Influence Spontaneous Alternation Performance: Relationships to Acetylcholine Release in the Hippocampus
}

\author{
Mark R. Stefani ${ }^{1}$ and Paul E. Gold ${ }^{2}$ \\ ${ }^{1}$ Department of Psychiatry, Yale University School of Medicine, Veterans Affairs Medical Center, West Haven, Connecticut \\ 06516, and 2Department of Psychology, University of Illinois at Urbana-Champaign, Champaign, Illinois 61820
}

One mechanism by which administration of glucose enhances cognitive functions may be by modulating central ATP-sensitive potassium (K-ATP) channels. K-ATP channels appear to couple glucose metabolism and neuronal excitability, with channel blockade increasing the likelihood of neurosecretion. The present experiment examined the effects of glucose and the direct K-ATP channel modulators glibenclamide and lemakalim on spontaneous alternation performance and hippocampal ACh release. Rats received either artificial CSF vehicle or vehicle plus drug for two consecutive 12 min periods via microdialysis probes ( $3 \mathrm{~mm}$; flow rate of $2.1 \mu \mathrm{l} / \mathrm{min}$ ) implanted in the left hippocampus. During the second $12 \mathrm{~min}$ period, rats were tested for spontaneous alternation performance. Dialysate was simultaneously collected for later analysis of ACh content. Both glucose $(6.6 \mathrm{~mm})$ and glibenclamide $(100 \mu \mathrm{M})$ significantly increased alternation scores compared with those of controls. Conversely, lemakalim (200 $\mu \mathrm{M})$ significantly reduced alterna- tion scores relative to those of controls. Simultaneous administration of lemakalim with either glucose or glibenclamide resulted in alternation scores not significantly different from control values. All drug treatments enhanced hippocampal ACh output relative to control values. The results demonstrate that K-ATP channel modulators influence behavior when administered directly into the hippocampus, with channel blockers enhancing and openers impairing spontaneous alternation performance, thus supporting the hypothesis that glucose enhances memory via action at central K-ATP channels. That lemakalim, as well as glibenclamide and glucose, increased hippocampal ACh output suggests a dissociation between the effects of K-ATP channel modulators on behavior and hippocampal ACh release.

Key words: memory; K-ATP channels; spatial memory; glucose; glibenclamide; lemakalim; hippocampus; acetylcholine
Systemic administration of D-glucose at doses that produce modest, physiological elevations in blood glucose levels enhance memory for aversive, appetitive, spatial, and habituation tasks in rodents (Messier and White, 1984, 1987; Gold, 1986; Messier and Destrade, 1988; Kopf and Baratti, 1994, 1996; Ragozzino et al., 1996). Central glucose injections also enhance aversive and spatial learning and memory (Lee et al., 1988; Ragozzino et al., 1998; Stefani et al., 1999). Because glucose freely crosses the bloodbrain barrier, the observation that central and systemic glucose administration produce similar behavioral effects suggests a central mechanism of action. Consistent with this view, recent findings indicate that extracellular glucose levels in the hippocampus decrease during maze testing (McNay et al., 2000a,b).

Glucose may enhance memory by modulating the conductance of central ATP-sensitive potassium (K-ATP) channels. K-ATP channel conductance is decreased by increases in intracellular ATP levels and by sulfonylurea-class drugs such as glibenclamide and is increased by decreases in intracellular ATP concentration

\footnotetext{
Received May 16, 2000; revised Oct. 2, 2000; accepted Oct. 12, 2000.

This work was supported by National Institute on Aging Grant AG07648, National Institute of Neurological Disorders and Stroke Grant NS32914, United States Department of Agriculture Grant 00-35200-9059, and a research grant from the Alzheimer Association. M.R.S. was supported by National Institute of Mental Health predoctoral fellowship Grant MH11057. We thank SmithKline Beecham Pharmaceuticals for their donation of lemakalim and Thomas Fries for his technical assistance.

Correspondence should be addressed to Dr. Paul E. Gold, Department of Psychology, University of Illinois at Urbana-Champaign, 603 East Daniel Street, Champaign, IL 61820. E-mail: pgold@uiuc.edu.

Copyright (C) 2001 Society for Neuroscience 0270-6474/01/010609-06\$15.00/0
}

and by drugs such as lemakalim. Decreases in K-ATP channel conductance increase cellular sensitivity to depolarizing stimuli, increasing the likelihood of stimulus-evoked neurotransmitter release. K-ATP channels have been suggested as a mechanism coupling fluctuations in neuronal glucose metabolism to neurosecretion (Amoroso et al., 1990), with hyperglycemia leading to enhanced, and hypoglycemia to reduced, transmitter release.

Direct K-ATP channel modulators such as glibenclamide and lemakalim have been shown to affect neurotransmitter release both in vitro (Amoroso et al., 1990) and in vivo (During et al., 1995; Tanaka et al., 1995). Little attention has thus far been paid, however, to the effects of K-ATP channel modulators on neurotransmitter release and memory in behaving animals. In one such experiment, glibenclamide attenuated impairments of inhibitory (passive) avoidance memory produced by intraventricular injections of various K-ATP channel openers (Ghelardini et al., 1998). Results from our laboratory have shown that intraseptal injections of glibenclamide, such as glucose, enhance spontaneous alternation performance and antagonize impairments in alternation scores produced by the K-ATP channel openers morphine and galanin (Stefani and Gold, 1998; Stefani et al., 1999). Spontaneous alternation performance, which is sensitive to inter-arm delay intervals, to the number of maze arms, and to spatial cues, has been used for many years to assess spatial working memory and rates of forgetting (Zornetzer et al., 1982; Tako et al., 1988; Ragozzino et al., 1996; Caston et al., 1997; Talley et al., 2000; McNay et al., 2000a,b) (cf. Dember and Richman, 1989).

The present experiment examined the effects on spatial mem- 
ory testing of direct intrahippocampal administration of glibenclamide and lemakalim to close and open, respectively, K-ATP channels. The experiment also examined interactions between lemakalim and glibenclamide and glucose on maze performance. In addition, because regulation of ACh release is a possible target for the actions of glucose (Messier et al., 1990; Kopf and Baratti, 1994, 1996; Ragozzino et al., 1994a,b, 1996, 1998; Ragozzino and Gold, 1995), the present experiment examined ACh output during spatial memory testing and drug administration.

\section{MATERIALS AND METHODS}

Subjects. Male Sprague Dawley rats (Hilltop Laboratory, Dublin, VA) weighing $275-300 \mathrm{gm}$ at time of arrival were used as subjects. Thirty-six rats in total were used for the experiments described below. Rats were housed individually in a room maintained on a $12 \mathrm{hr}$ light/dark cycle (lights on at 7:00 A.M.) and had ad libitum access to food and $\mathrm{H}_{2} \mathrm{O}$. Housing and care of laboratory animals were in compliance with institutional and federal regulations.

Surgery. Rats were anesthetized with sodium pentobarbital $(50 \mathrm{mg} / \mathrm{kg}$, i.p.) $20 \mathrm{~min}$ after the administration of atropine sulfate (108 $\mu \mathrm{g} / \mathrm{rat}$, i.p.). Plastic guide cannulas (model CMA/12; Carnegie Medicin, Stockholm, Sweden) directed at the hippocampus were implanted using stereotaxic coordinates derived from the atlas of Pellegrino et al. (1979) $(3.8 \mathrm{~mm}$ caudal to bregma, $5.0 \mathrm{~mm}$ lateral to midline, $4.6 \mathrm{~mm}$ ventral from skull surface). Jeweler's screws were placed in the skull around the cannulas, and the cannulas were secured to these screws with dental acrylic (Plastics One, Roanoke, VA). Rats were permitted a 1 week recovery period, during which they were handled daily.

The tangential cannula placement, most often including portions of both dorsal and ventral hippocampus, was needed to ensure that the dialysis probe was confined to the hippocampus. Although functional differences exist between dorsal and ventral hippocampus (Hock and Bunsey, 1998; Moser and Moser, 1998; Richmond et al., 1999), there is clear evidence that the ventral hippocampus, like the dorsal hippocampus, is important for mnemonic functions (Poucet et al., 1991; Poucet and Buhot, 1994; Lorenzini et al., 1997). Importantly, there is considerable evidence indicating that the ventral, as well as dorsal, hippocampus is involved in spatial memory and that pharmacological modulation of ventral hippocampal function influences performance on tests of spatial memory (Poucet et al., 1991; Kim and Levin, 1996; Oegren et al., 1998; Levin et al., 1999).

Microdialysis. On the day of experiment, a microdialysis probe (CMA/ 12; Carnegie Medicin) with a dialysis surface $3 \mathrm{~mm}$ in length was inserted through the guide cannula and perfused continuously with vehicle solution at a rate of $2.1 \mu \mathrm{l} / \mathrm{min}$ for a period sufficient to achieve stable baseline ACh output ( $\sim 1 \mathrm{hr})$. Subsequent samples were collected at $12 \mathrm{~min}$ intervals ( $25 \mu \mathrm{l}$ sample volume). The first three samples served to establish a baseline level of ACh output. Rats received either the vehicle solution or vehicle plus drug during samples 4 and 5 and then vehicle solution again for samples $6-8$. Spontaneous alternation testing was conducted during the administration of sample 5. The vehicle solution was composed of (in mM): $154 \mathrm{NaCl}, 3 \mathrm{KCl}, 1.5 \mathrm{CaCl}_{2}, 1.0 \mathrm{MgCl}_{2}$, $2.0 \mathrm{NaH}_{2} \mathrm{PO}_{4}, 2.0 \mathrm{Na}_{2} \mathrm{HPO}_{4}$, and 2.0 glucose, $\mathrm{pH}$ 7.4. The vehicle solution also contained the acetylcholinesterase inhibitor neostigmine $(1.0 \mu \mathrm{M})$ to enhance ACh recovery and dimethylsulfoxide (DMSO) $(0.5 \% \mathrm{v} / \mathrm{v})$ to ensure solubilization of the glibenclamide and lemakalim. Lemakalim was provided as a gift by SmithKline Beecham Pharmaceuticals (King of Prussia, PA). All other reagents were purchased from Sigma (St. Louis, MO).

Drug treatments were administered by reverse dialysis, with the vehicle modified to include (final concentrations) $6.6 \mathrm{~mm}$ glucose, $100 \mu \mathrm{M}$ glibenclamide, and $200 \mu \mathrm{M}$ lemakalim. Glucose and glibenclamide concentrations were selected on the basis of past findings (Ragozzino et al., 1998; Stefani et al., 1999) and pilot studies; the lemakalim concentration was selected on the basis of pilot studies indicating that this was the lowest concentration that reliably impaired spontaneous alternation scores.

Behavioral procedure. Each rat was assigned pseudorandomly to two of the following treatment groups: vehicle control (VEH), glucose (GLC), glibenclamide (GLB), lemakalim (LEM), or lemakalim plus glucose (LEM/GLC), such that no rat received the same treatment twice. The first test session was separated from the date of surgery by a 1 week interval, as was the second test session from the first. A group examining the combined effects of glibenclamide and lemakalim (LEM/GLB) was later added to the study; these rats were tested only once. Spontaneous alternation testing was conducted by placing the rat on the center platform of a four-arm radial maze (plus maze) and allowing $12 \mathrm{~min}$ of unimpeded exploration. The number and sequence of arm entries were recorded for calculation of a percent alternation score. An alternation consisted of 4 different arm choices of 5 consecutive arm entries. A 4/5 alternation score was computed by dividing the number of observed alternations in overlapping quintuplets by the number of possible alternations and multiplying the quotient by 100 .

The plus maze was composed of four arms joined to an central platform. Each arm was $55 \times 10 \times 12 \mathrm{~cm}$. The central platform was 25 $\mathrm{cm}$ across. The floor and walls of the central platform and the floors of the arms were made of gray-painted wood. The walls of the arms were made of poster board and were periodically changed to prevent the accumulation of odors. The maze floor was washed with ethanol $(70 \%$ $\mathrm{v} / \mathrm{v}$ ) between animals.

Acetylcholine analysis. Dialysate samples $(20 \mu \mathrm{l})$ were analyzed for ACh content by reverse-phase HPLC coupled with an enzymatic assay system and electrochemical detector. Acetylcholine and choline were separated on a reverse-phase column (Chromspher $5 \mathrm{C} 18 ; 100 \times 3 \mathrm{~mm}$; Chrompack, Middelburg, The Netherlands). A post-column enzyme reactor unit containing acetylcholinesterase (EC 3.1.1.7; Sigma type VI-S) and choline oxidase (EC 1.1.3.17; Sigma) converted ACh ultimately to betaine and $\mathrm{H}_{2} \mathrm{O}_{2}$. Oxidation of the $\mathrm{H}_{2} \mathrm{O}_{2}$ was detected electrochemically using a platinum working electrode held at $+525 \mathrm{mV}$ relative to a silver chloride reference electrode. Acetylcholine peaks were quantified by comparison with peaks generated by ACh standards prepared at the time of each experiment. The lower detection limit of the system for ACh was $50 \mathrm{fmol}$. The mobile phase contained (in mM): $0.2 \mathrm{~K}_{2} \mathrm{HPO}_{4}, 1.0$ tetramethylammonium hydroxide, and 0.3 EDTA, pH 8.0, with $0.005 \%$ Kathon CG to retard bacterial growth. Mobile phase flow rate was $0.6 \mathrm{ml} / \mathrm{min}$.

Histology. After behavioral testing, the rats were killed with an overdose of sodium pentobarbital and perfused with solutions of $0.9 \% \mathrm{NaCl}$ and $10 \%$ formalin. Their brains were removed and stored in a $30 \%$ sucrose $-10 \%$ formalin solution until sectioning. Serial sections $(40-\mu \mathrm{m}-$ thick) through the hippocampal area were mounted on glass slides and stained with cresyl violet. Stained sections were evaluated for accuracy of cannula placement. Animals with cannula placements outside of the hippocampus were excluded from subsequent analysis. Figure 1 illustrates the area of the hippocampus in which cannula placements were accepted for inclusion in behavioral and neurochemical data analyses.

Statistical analysis. Behavioral data were analyzed by one-way, between-subject ANOVA with Fisher's post hoc tests. Microdialysis data were analyzed by first converting raw ACh output scores for each subject to a percentage of their respective baseline ACh output. The baseline was calculated as the mean of the three samples preceding drug treatment. Within-subject comparisons of ACh output were by repeated measures ANOVA with Bonferroni correction. Between-group comparisons of ACh output for select samples were by Student's $t$ test.

\section{RESULTS}

\section{Histology}

For all rats included here, dialysis probes were located entirely within the hippocampal formation (Fig. 1). The majority of the length of the dialysis membrane was located in the medial dentate gyrus and CA3 regions. The bottom of the dialysis membrane extended in some cases as ventral as the margin of the hippocampus and ventral subiculum.

\section{Spontaneous alternation behavior}

As shown in Figure 2, intrahippocampal infusions of K-ATP channel modulators significantly altered spontaneous alternation scores relative to those of vehicle-injected controls $\left(F_{(5,39)}=\right.$ 17.84; $p<0.001)$. Glucose significantly increased spontaneous alternation scores relative to those of vehicle-infused control animals $(p<0.05)$, in agreement with previous findings (Ragozzino et al., 1998). Intrahippocampal infusion of the direct K-ATP channel blocker glibenclamide $(100 \mu \mathrm{M})$ significantly increased spontaneous alternation scores relative to those of vehicle-infused control animals and glucose-infused animals 


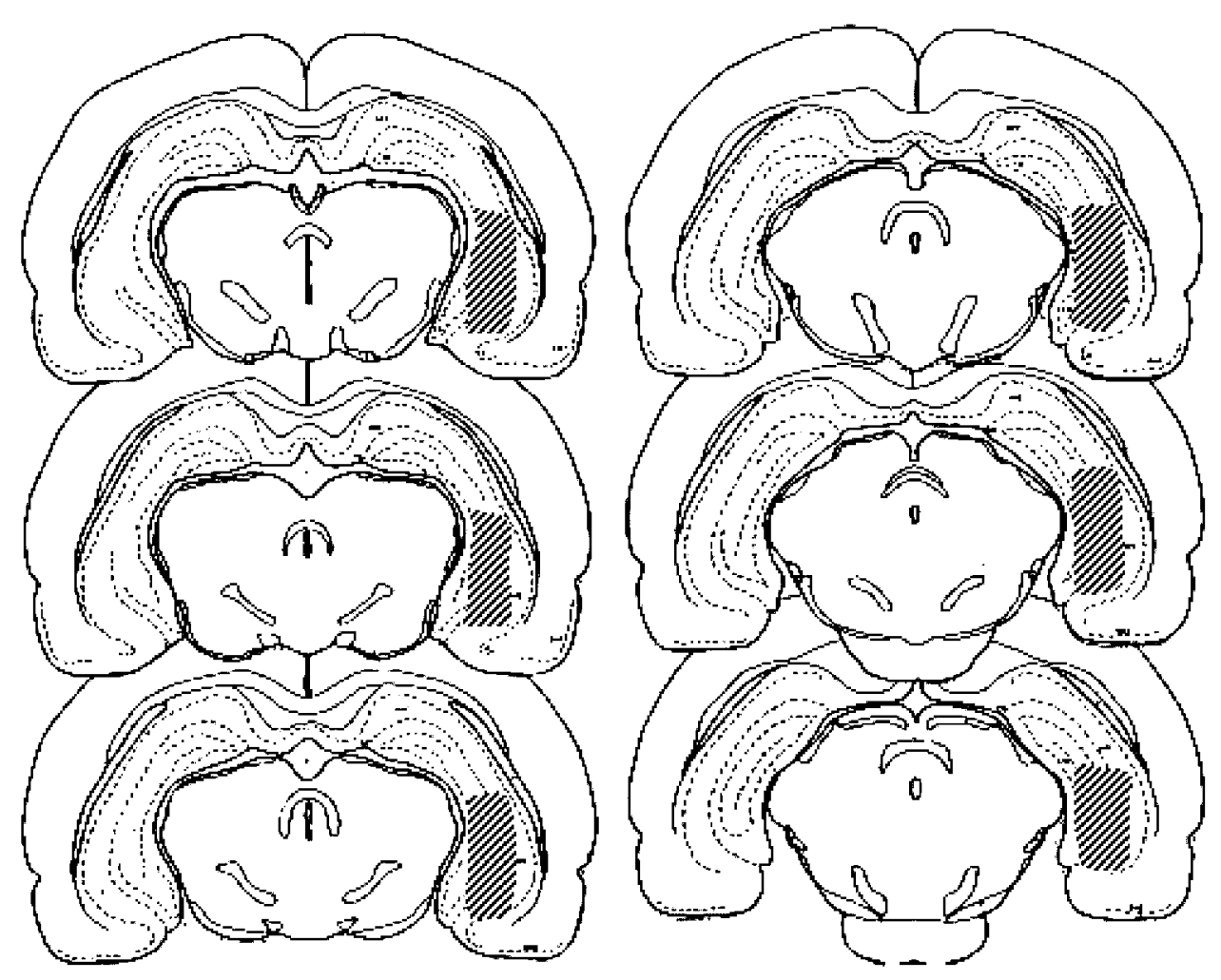

Figure 1. The shaded areas represent the extent of acceptable intrahippocampal probe placements. Animals with probe placements outside of the shaded areas were excluded from analysis. The atlas plates shown are adapted from the stereotaxic atlas of Pellegrino et al. (1979).

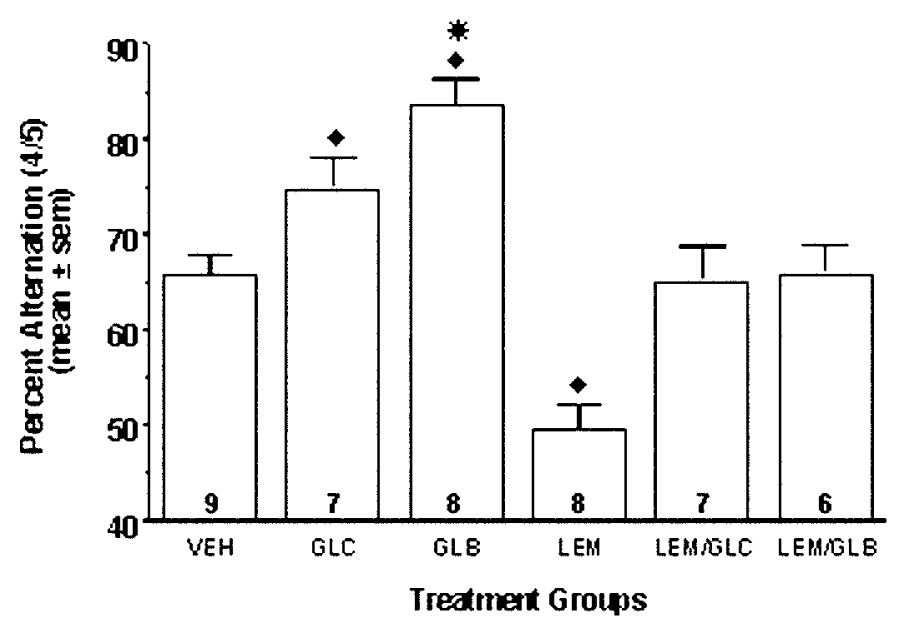

Figure 2. Effects on alternation scores of intrahippocampal injections of glucose, glibenclamide, lemakalim, and lemakalim plus glucose or glibenclamide. Infusion of glucose $(6.6 \mathrm{~mm})$ or glibenclamide $(100 \mu \mathrm{M})$ significantly enhanced spontaneous alternation scores above those of vehicleinfused controls. Infusion of lemakalim $(200 \mu \mathrm{M})$ significantly impaired alternation scores relative to those of controls. Coadministration of either glucose or glibenclamide and lemakalim resulted in alternation scores not significantly different from those of controls. $p<0.05$ versus $\mathrm{VEH}$, LEM/GLC, and LEM/GLB; * $p<0.05$ versus GLC. Numbers within bars indicate the number of rats per group included in data analysis.

$(p<0.05)$. Conversely, intrahippocampal infusion of the direct K-ATP channel opener lemakalim significantly decreased spontaneous alternation scores relative to vehicle-injected control animals $(p<0.05)$. Coadministration of either glucose or glibenclamide with lemakalim attenuated the lemakalim-induced re- duction in spontaneous alternation scores; alternation scores in rats treated with glucose plus lemakalim or glibenclamide plus lemakalim did not differ from those of vehicle-inf used rats $(p>$ $0.05)$.

No significant between-group differences in the number of arm entries were observed $\left(F_{(5,39)}=0.44 ; p=0.82\right)$. The number of arm entries for each group were as follows (mean $\pm \mathrm{SE}$ ): $\mathrm{VEH}$, $26.0 \pm 1.6$; GLC, $25.3 \pm 1.1$; GLB, $25.8 \pm 2.9$; LEM, $29.6 \pm 2.5$; LEM/GLC, $25.7 \pm 2.7$; and LEM/GLB, $26.0 \pm 3.7$. There was no significant effect of treatment order on alternation performance $(p=0.34)$ or number of arm entries $(p=0.15)$.

\section{Hippocampal acetylcholine output}

Spontaneous alternation behavior increased hippocampal ACh output in vehicle-infused rats over the baseline average $(p<$ 0.05 ) (Fig. 3A, sample 5). Treatment with glibenclamide, lemakalim, or lemakalim plus glucose in conjunction with maze performance significantly increased hippocampal ACh output above the level observed for vehicle-inf used controls ( $p$ values $<$ 0.05 ) (Fig. 3A, sample 5). Hippocampal ACh output was still significantly elevated compared with baseline levels during the 12 min time period immediately after spontaneous alternation behavior for all groups except glibenclamide and lemakalim plus glibenclamide $(p$ values $<0.05)$ (Fig. 3A, sample 6). After removal of the rats from the maze to the holding cage, hippocampal ACh levels returned toward baseline levels (Fig. 3A, samples 6-8). Hippocampal ACh output after spontaneous alternation testing returned to stable levels not significantly different from baseline levels in all groups except VEH; ACh output during sample 8 was significantly lower than baseline levels $(p<0.05)$ (Fig. $3 A$, sample 8 ). There was no significant correlation between hippocampal ACh levels and motor activity as measured by the 

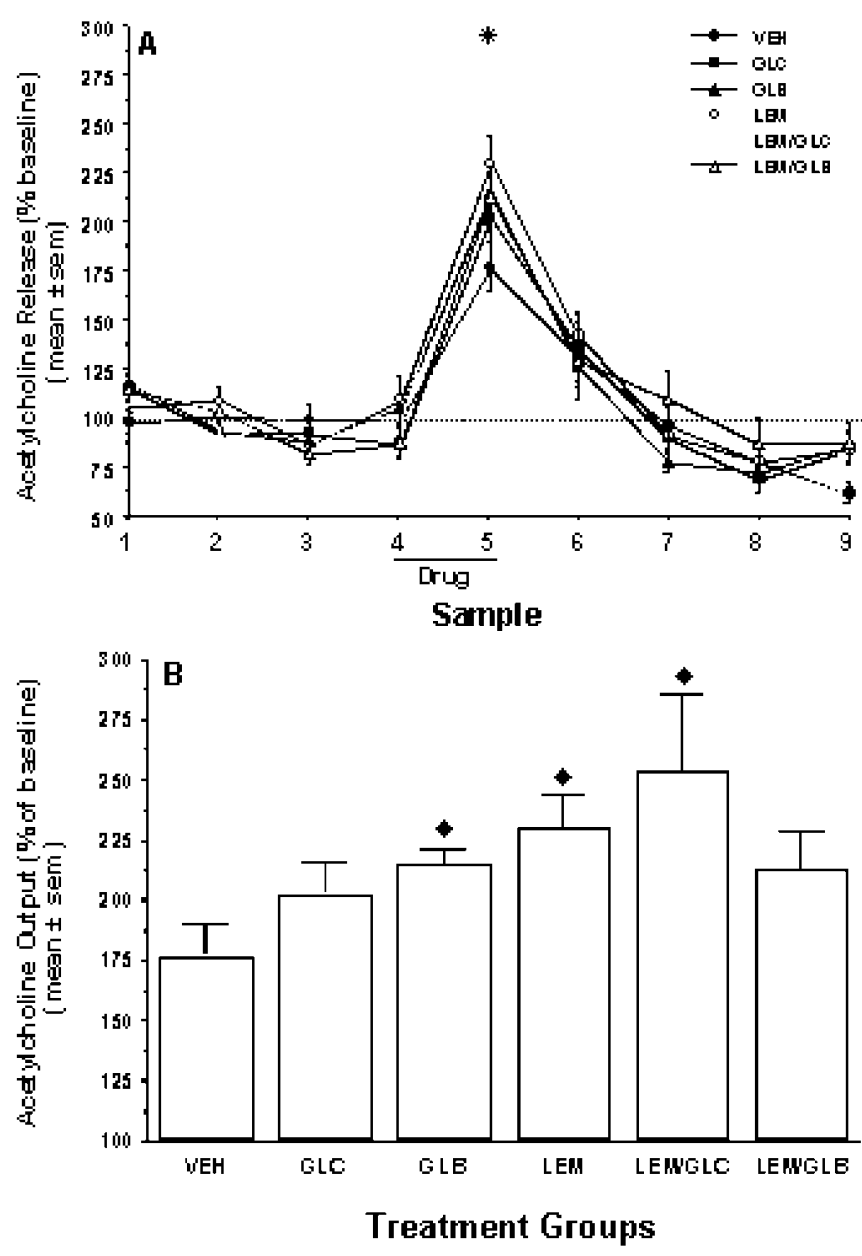

Figure 3. Effects of behavioral testing and drug treatments on extracellular ACh levels in the hippocampus. Drugs were administered immediately before the start of sample 4 collection. Alternation tests were conducted during sample 5. A, Time course of changes in ACh output in the hippocampus. Note that spontaneous alternation testing resulted in significant increases in hippocampal ACh output relative to baseline levels in all groups. For sample $5,{ }^{*} p$ values $<0.05$ versus baseline for all groups. $B$, ACh output in hippocampus during behavioral testing. Note that ACh increased by $75 \%$ in vehicle controls. All drug treatments resulted in further increases in hippocampal ACh output, with increases after GLB, LEM, and LEM/GLC. $p<0.05$ versus VEH.

number of arm entries during spontaneous alternation testing $(r=-0.241)$.

The baseline levels of ACh for each group were as follows(mean \pm SEM picomoles of ACh per $25 \mu \mathrm{l}$ sample, corrected for probe recovery): $\mathrm{VEH}, 5.1 \pm 0.9$; GLC, $5.0 \pm 0.6$; GLB, $5.0 \pm$ 0.6; LEM, $4.3 \pm 0.4 ; \mathrm{LEM} / \mathrm{GLC}, 3.6 \pm 0.6$; and LEM/GLB, $4.3 \pm 0.5$. Baseline levels of ACh did not vary significantly between groups $\left(F_{(5,35)}=2.49 ; p=0.42\right)$. Furthermore, the baseline levels of $\mathrm{ACh}$ reported here are in the range of those previously reported by this laboratory for hippocampal dialysis using perfusion fluid that did not contain the solubilizing agent DMSO (Ragozzino et al., 1994a, 1998). Thus, the DMSO used in the present study appears to have had negligible, if any, effect on basal ACh release.

\section{DISCUSSION}

Unilateral hippocampal dialysis with a solution containing either $6.6 \mathrm{~mm}$ glucose or $100 \mu \mathrm{M}$ glibenclamide significantly enhanced spontaneous alternation scores relative to rats dialyzed with vehicle containing $2 \mathrm{~mm}$ glucose. These present findings are consistent with previous data showing that administration of glucose to the hippocampus via microdialysis enhances spontaneous alternation scores (Ragozzino et al., 1998) and extend these findings by showing that hippocampal administration of glibenclamide, which like glucose blocks K-ATP channels, also increases spontaneous alternation scores. Conversely, hippocampal dialysis with the K-ATP channel opener lemakalim (200 $\mu \mathrm{M})$ significantly impaired spontaneous alternation performance relative to that of control animals. Both glucose and glibenclamide attenuated the performance-impairing effect of lemakalim.

The glucose metabolic product ATP and sulfonylurea-class drugs, such as glibenclamide, antagonize the effects of lemakalim on K-ATP channel function in vitro (Stanford and Lacey, 1996; Lauritzen et al., 1997, Schwanstecher and Bassen, 1997); the present results suggest that this antagonism extends as well to effects on behavior. These results are also consistent with findings obtained with intraseptal injections of glucose or glibenclamide, which enhance spontaneous alternation scores and also attenuate impairments in spontaneous alternation scores induced by the indirectly acting K-ATP channel openers morphine and galanin (Ragozzino et al., 1992; Ragozzino and Gold, 1995; Stefani and Gold, 1998; Stefani et al., 1999). Furthermore, the enhancing effects of glucose and glibenclamide on spontaneous alternation behavior are additive (Stefani et al., 1999).

Both the septal area and the hippocampus abundantly express K-ATP channels (Mourre et al., 1990, 1991), and hippocampal K-ATP channels appear to be influenced by behavioral manipulations (Fellows et al., 1993). Thus, neurons at the origin and termination of the septohippocampal pathway are potentially subject to regulation by local fluctuations in extracellular glucose levels. Decreases in extracellular glucose concentrations have been observed recently during alternation testing on a four-arm maze (McNay et al., 2000a,b), providing additional evidence that glucose availability might regulate processes important for memory processing, perhaps via K-ATP channels.

Spontaneous alternation behavior was used here as a measure of spatial working memory based on findings that performance on this task is sensitive to delay between arm choices, is dependent in part on extramaze cues, and that many potential alternative interpretations of the drug-induced impairments, such as effects on turning bias rather than on memory, are inconsistent with previous findings obtained with this same task in our laboratory and many others (Tako et al., 1988; Beracochea et al., 1989; Lalonde et al., 1993; Vaillend et al., 1995; Ragozzino et al., 1996; Caston et al., 1997; Talley et al., 2000) (cf. Dember and Richman, 1989). Also, in the present experiment, there were no significant between-group differences in the number of arm entries during the 12 min spontaneous alternation trial, suggesting that the behavioral effects of the drug treatments were attributable to an influence on mnemonic processes rather than an effect on general motor activity or to differences in inter-arm, i.e., intertrial, interval. A mnemonic effect of K-ATP channel modulators is also supported by the finding that intraventricular administration of several K-ATP channel openers impairs memory for inhibitory avoidance training and that glibenclamide attenuates these impairments (Ghelardini et al., 1998).

The present study also examined the effects of K-ATP channel modulators on hippocampal ACh release in conjunction with spontaneous alternation testing. Substantial evidence, assessed thus far primarily in the hippocampus, indicates that glucose may 
enhance memory by augmenting cholinergic functions (Messier et al., 1990; Durkin et al., 1992; Ragozzino et al., 1994a; Micheau et al., 1995). Maze performance per se significantly increases hippocampal ACh output above baseline levels; acute glucose administration further increases this behavior-dependent ACh release (Ragozzino et al., 1994a, 1996, 1998; present data). In the present study, both glucose and glibenclamide administration augmented the increase in hippocampal ACh outputs seen in controls. Unlike previous studies, however, the increase after glucose administration was not significant, although it approached significance. One difference between the previous and present studies is that vehicle-infused rats in the present studies had a higher behavior-induced increase in ACh output, as well as higher control alternation scores compared with those in past studies that used the same behavioral apparatus (Ragozzino et al., 1996, 1998).

In previous studies, glucose attenuated decreases in hippocampal ACh release caused by systemic and intraseptal injections of the opioid receptor agonist and indirect K-ATP channel opener morphine (Ragozzino et al., 1994a; Ragozzino and Gold, 1995); these neurochemical results paralleled attenuation by glucose of morphine-induced impairments in alternation performance. In contrast, the present findings show that hippocampal dialysis with lemakalim, alone or in combination with glucose or glibenclamide, increased behavior-dependent hippocampal ACh release above control levels, with the highest levels of hippocampal $\mathrm{ACh}$ release seen in the groups receiving lemakalim alone or in combination with glucose. Thus, although most conditions resulted in relationships between ACh release and memory consistent with previous findings, there was a dissociation between spontaneous alternation scores and hippocampal ACh release after administration of lemakalim. The different findings may reflect different and perhaps multiple sites of action of morphine and lemakalim with respect to ACh participation in memory. One interpretation of the findings is that morphine acts before the contribution of ACh to memory processing and that lemakalim acts at a step beyond the contribution of ACh to memory processing. According to this interpretation, ACh release would be associated with learning and memory for manipulations acting before or at cholinergic mechanisms but would not be associated with learning and memory for manipulations acting thereafter. Note, however, that this is a description of perhaps the simplest condition. More likely, multiple neurotransmitters contribute to memory processing in parallel. Consistent with the possibility that regulation of K-ATP channels may influence many neurotransmitters are the effects seen on GABA, dopamine, serotonin, and glutamate (Mourre et al., 1991; Zetterström et al., 1991; During et al., 1995; Tanaka et al., 1995; Lee et al., 1996). Of these neurotransmitter systems, glucose effects on memory have been examined and observed as interactions with drugs directed at GABA and glutamate receptors (Walker and Gold, 1992; Lennartz and Gold, 1995; Parent and Gold, 1997; Parent et al., 1997). If the effects on memory of glucose and K-ATP channel modulators are through multiple neurochemical systems, characterization of the relative contributions of these different neurotransmitters to memory processing will require examination of release of multiple neurotransmitters while rats are performing memory tasks under many different drug conditions.

In conclusion, the present data demonstrate that central administration of K-ATP channel modulators influence spatial memory, with channel blockers enhancing and channel openers impairing spontaneous alternation behavior. Thus, the data pro- vide further support for the hypothesis that glucose enhances memory via modulation of K-ATP channel conductance. Because $\mathrm{K}$-ATP channel activity is regulated by intracellular ATP concentrations (Panten et al., 1996), K-ATP channels may couple neurosecretion to neuronal glucose metabolism (Amoroso et al., 1990). According to this view, increases in glucose may enhance stimulus-evoked transmitter release, thereby providing an attractive mechanism by which peripheral and central glucose administration enhances cognitive functions. Further experiments examining the effects of glucose and direct K-ATP channel modulators on behavior-dependent neurotransmitter release will clarify the system(s) through which glucose exerts its mnemonic effects.

\section{REFERENCES}

Amoroso S, Schmid-Antomarchi H, Fosset M, Lazdunski M (1990) Glucose, sulfonylureas, and neurotransmitter release: role of ATP-sensitive $\mathrm{K}^{+}$channels. Science 247:852-854

Beracochea DJ, Tako AN, Jaffard R (1989) Accelerated rates of forgetting of spatial information during aging and long-term ethanol consumption in mice: evidence for two distinct forms of amnesia. Psychobiology 17:358-362.

Caston J, Vasseur F, Delhaye-Bouchaud N, Mariani J (1997) Delayed spontaneous alternation in intact and cerebellectomized control and lurcher mutant mice: differential role of cerebellar cortex and deep cerebellar nuclei. Behav Neurosci 111:214-218.

Dember WN, Richman CL (1989) Spontaneous alternation behavior, p 212. New York: Springer.

During MJ, Leone P, Davis KE, Kerr D, Sherwin RS (1995) Glucose modulates rat substantia nigra GABA release in vivo via ATP-sensitive potassium channels. J Clin Invest 95:2403-2408.

Durkin TP, Messier C, de Boer P, Westerink BHC (1992) Raised glucose levels enhance scopolamine-induced acetylcholine overflow from the hippocampus: an in vivo microdialysis study in the rat. Behav Brain Res 49:181-188.

Fellows LK, Boutelle MG, Fillenz M (1993) ATP-sensitive potassium channels and local energy demands in the rat hippocampus: an in vivo study. J Neurochem 61:949-954.

Ghelardini C, Galeotti N, Bartolini A (1998) Influence of potassium channel modulators on cognitive processes in mice. Br J Pharmacol 123:1079-1084.

Gold PE (1986) Glucose modulation of memory storage processing. Behav Neural Biol 45:342-349.

Hock Jr BJ, Bunsey MD (1998) Differential effects of dorsal and ventral hippocampal lesions. J Neurosci 18:7027-7032.

Kim JS, Levin ED (1996) Nicotinic, muscarinic and dopaminergic actions in the ventral hippocampus and the nucleus accumbens: effects on spatial working memory in rats. Brain Res 725:231-240.

Kopf SR, Baratti CM (1994) Memory-improving actions of glucose: involvement of a central cholinergic muscarinic mechanism. Behav Neural Biol 62:237:243

Kopf SR, Baratti CM (1996) Effects of posttraining administration of glucose on retention of a habituation response in mice: participation of a central cholinergic mechanism. Neurobiol Learn Mem 65:253-260.

Lalonde R, Joyal CC, Cote C, Botez MI (1993) Delayed spontaneous alternation in lurcher mutant mice. Psychobiology 21:139-141.

Lauritzen I, de Weille JR, Lazdunski M (1997) The potassium channel opener (-)-cromakalim prevents glutamate-induced cell deather in hippocampal neurons. J Neurochem 69:1570-1579.

Lee K, Dixon AK, Rowe ICM, Ashford MLJ, Richardson PJ (1996) The high-affinity sulphonylurea receptor regulates KATP channels in nerve terminals of the rat motor cortex. J Neurochem 66:2562-2571.

Lee MK, Graham SN, Gold PE (1988) Memory enhancement with post-training intraventricular glucose injections in rats. Behav Neurosci 4:591-595.

Lennartz RC, Gold PE (1995) Glucose does not reverse impairments on spontaneous alternation induced by the noncompetitive NMDA antagonist MK-801. Neurobiol Learn Mem 63:107-110.

Levin ED, Christopher NC, Weaver T, Moore J, Brucato F (1999) Ventral hippocampal ibotenic acid lesions block chronic nicotineinduced spatial working memory improvement in rats. Cognit Brain Res 7:405-410.

Lorenzini CGA, Baldi E, Bucherelli C, Sacchetti B, Tassoni G (1997) Role of ventral hippocampus in acquisition, consolidation and retrieval of rat's passive avoidance response memory trace. Brain Res 768:242-248.

McNay EC, Fries TM, Gold PE (2000a) Decreases in rat extracellular hippocampal glucose concentration associated with cognitive demand during a spatial task. Proc Natl Acad Sci USA 97:2881-2885. 
McNay EC, McCarty RM, Gold PE (2000b) Fluctuations in glucose concentration during behavioral testing: dissociations both between brain areas and between brain and blood. Neurobiol Learn Mem, in press.

Messier C, Destrade C (1988) Improvement of memory for an operant response by post-training glucose in mice. Behav Brain Res 31:185-191.

Messier C, White NM (1984) Contingent and non-contingent actions of sucrose and saccharin reinforcers: effects on taste preference and memory. Physiol Behav 32:195-203.

Messier C, White NM (1987) Memory improvement by glucose, fructose, and two glucose analogs: a possible effect on peripheral glucose transport. Behav Neural Biol 48:104-127.

Messier C, Durkin T, Mrabet O, Destrade C (1990) Memory-improving action of glucose: indirect evidence for a facilitation of hippocampal acetylcholine synthesis. Behav Brain Res 39:135-143.

Micheau J, Messier C, Jaffard R (1995) Glucose enhancement of scopolamine-induced increase of hippocampal high-affinity choline uptake in mice: relation to plasma glucose level. Brain Res 685:99-104.

Moser M-B, Moser EI (1998) Functional differentiation in the hippocampus. Hippocampus 8:608-619.

Mourre C, Widmann C, Lazdunski M (1990) Sulfonylurea binding sites associated with ATP-regulated $\mathrm{K}^{+}$channels in the central nervous system: autoradiographic analysis of their distribution and ontogenesis, and of their localization in mutant mice cerebellum. Brain Res 519:29-43.

Mourre C, Widmann C, Lazdunski M (1991) Specific hippocampal lesions indicate the presence of sulfonylurea binding sites associated to ATP-sensitive $\mathrm{K}^{+}$channels both post-synaptically and on mossy fibers. Brain Res 540:340-344.

Oegren SO, Schoett PA, Kehr J, Yoshitake T, Misane I, Mannstroem P, Sandin J (1998) Modulation of acetylcholine and serotonin transmission by galanin. Relationship to spatial and aversive learning. In: Galanin: basic research discoveries and therapeutic implications (Hoekfelt T, Bartfai T, Crawley J, eds), p 469. New York: New York Academy of Sciences.

Panten U, Schwanstecher M, Schwanstecher C (1996) Sulfonylurea receptors and mechanism of sulfonylurea action. Exp Clin Endocrinol 104:1-9.

Parent MB, Gold PE (1997) Intra-septal infusions of glucose potentiate inhibitory avoidance deficits when co-inf used with the GABA agonist muscimol. Brain Res 748:317-320.

Parent MB, Laurey PT, Wilkness S, Gold PE (1997) Intra-septal infusions of muscimol impair spontaneous alternation performance: infusions of glucose into the hippocampus, but not the medial septum, reverse the deficit. Neurobiol Learn Mem 68:75-85.

Pellegrino LJ, Pellegrino AS, Cushman AJ (1979) A stereotaxic atlas of the rat brain. New York: Plenum.

Poucet B, Buhot M-C (1994) Effects of medial septal or unilateral hippocampal inactivations on reference and working spatial memory in rats. Hippocampus 4:315-321.

Poucet B, Herrmann T, Buhot M-C (1991) Effects of short-lasting inactivations of the ventral hippocampus and medial septum on long-term and short-term acquisition of spatial information in rats. Behav Brain Res 44:53-65.

Ragozzino ME, Gold PE (1995) Glucose injections into the medial septum reverse the effects of intraseptal morphine infusions on hippocampal acetylcholine output and memory. Neuroscience 68:981-988.

Ragozzino ME, Parker ME, Gold PE (1992) Spontaneous alternation and inhibitory avoidance impairments with morphine injections into the medial septum: attenuation by glucose administration. Brain Res 597:241-249.

Ragozzino ME, Wenk GL, Gold PE (1994a) Glucose attenuates a morphine-induced decrease in hippocampal acetylcholine output: an in vivo microdialysis study in rats. Brain Res 655:77-82.

Ragozzino ME, Arankowsky-Sandoval G, Gold PE (1994b) Glucose attenuates the effect of combined muscarinic-nicotinic receptor blockade on spontaneous alternation. Eur J Pharmacol 256:31-36.

Ragozzino ME, Unick KE, Gold PE (1996) Hippocampal acetylcholine release during memory testing in rats: augmentation by glucose. Proc Natl Acad Sci USA 93:4693-4698.

Ragozzino ME, Pal SN, Unick K, Stefani MR, Gold PE (1998) Modulation of hippocampal acetylcholine release and of memory by intrahippocampal glucose injections. J Neurosci 18:1595-1601.

Richmond MA, Yee BK, Pouzet B, Veenman L, Rawlins JNP, Feldon J, Bannerman DM (1999) Dissociating context and space within the hippocampus: effects of complete, dorsal, and ventral excitotoxic hippocampal lesions on conditioned freezing and spatial learning. Behav Neurosci 113:1189-1203.

Schwanstecher C, Bassen D (1997) KATP-channel on the somata of spiny neurones in caudate nucleus: regulation by drugs and nucleotides. Br J Pharmacol 121:193-198.

Stanford IM, Lacey MG (1996) Electrophysiological investigation of adenosine triphosphate-sensitive potassium channels in the rat substantia nigra pars reticulata. Neuroscience 74:499-509.

Stefani MR, Gold PE (1998) Intra-septal injections of glucose and glibenclamide attenuate galanin-induced spontaneous alternation performance deficits in the rat. Brain Res 813:50-56.

Stefani MR, Nicholson GM, Gold PE (1999) K-ATP channel blockade enhances spontaneous alternation performance in the rat: a potential mechanism for glucose-mediated memory enhancement. Neuroscience 93:557-563.

Tako AN, Beracochea DJ, Jaffard R (1988) Accelerated rate of forgetting of spatial information following mammillary-body lesions in mice: effects of context change on retention-test performance. Psychobiology $16: 45-53$.

Talley CEP, Kahn S, Alexander L, Gold PE (2000) Epinephrine fails to enhance performance of food-deprived rats on a delayed spontaneous alternation task. Neurobiol Learn Mem 73:79-86.

Tanaka T, Yoshida M, Yokoo H, Mizoguchi K, Tanaka M (1995) The role of ATP-sensitive potassium channels in striatal dopamine release: an in vivo microdialysis study. Pharmacol Biochem Behav 52:831-835.

Vaillend C, Rendon A, Misslin R, Ungerer A (1995) Influence of dystrophin-gene mutation on mdx mouse behavior. I. Retention deficits at long delays in spontaneous alternation and bar-pressing tasks. Behav Genet 25:569-579.

Walker DL, Gold PE (1992) Impairment of spontaneous alternation performance by an NMDA antagonist: attenuation with non-NMDA treatments. Behav Neural Biol 58:69-71.

Zetterström T, Pei Q, Finn D, Grahame-Smith DG (1991) Increased brain DA and 5-HT release produced by inhibition of ATP sensitive $\mathrm{K}^{+}$channels: in vivo and in vitro studies. In: Monitoring molecules in neuroscience: Proceedings of the 5th International Conference on In Vivo Methods (Rollema H, Westerink B, Drijhout WJ, eds), pp 377380. Groningen, The Netherlands: University Centre for Pharmacy.

Zornetzer SF, Thompson R, Rogers J (1982) Rapid forgetting in aged rats. Behav Neur Biol 36:49-60. 